\title{
Addressing Workforce Needs by Disrupting Traditional Industry-Higher Education Relations: The Case of El Salvador1
}

\author{
Richard A. Navarro
}

California State Polytechnic University, USA

Estera Barbarasa

Independent Consultant, Economic Development Specialist

Ami Thakkar

RTI International, USA

\section{Corresponding author:}

Richard A. Navarro, California State Polytechnic University Pomona - Education, 3801 W. Temple Avenue Pomona, CA 91768, USA. Email: ranavarro@cpp.edu

\begin{abstract}
The authors analyze the recent undertaking in El Salvador to establish an innovative model of industry-higher education clusters that would facilitate collaboration between academia and the private sector - sectors that traditionally had not worked together because of historical distrust - to develop the skilled workforce needed for the country's future economic growth. Under the USAID Higher Education for Economic Growth project implemented by RTI International, clusters were put forth as the platform for building sustainable partnerships between industry and higher education - facilitating dialogue, stepping into each other's world and collaboration on curriculum planning, student internships and applied research. The clusters have pushed Salvadoran
\end{abstract}


universities to become more agile organizations, able to pivot to meet industry skills demands, and industries to recognize universities' contributions to the creation of value for increased productivity. The El Salvador case is a collaborative multi-stakeholder model to meet workplace requirements for high-growth industries in low- to middleincome economies.

\section{Keywords:}

University-industry collaboration, workforce development, skills gap, industryuniversity clusters, college to career readiness, agile organizations

The mismatch between the supply of workers' skills and the demands of the new economy has led to calls for higher education to improve its labor market relevance and to engage more closely with employers. In the UK the report Prosperity for All in the Global Economy: World Class Skills (Leitch, 2006) led to new resources for and interest in stimulating university-industry collaboration (UIC). In the USA the Obama Administration took the lead in shining a spotlight on the connection between increasing the number of Americans with a college degree and closing the skills gap, particularly in science, technology engineering and mathematics (STEM) fields. President Obama committed the USA to having the highest proportion of college graduates in the world by 2020 (The White House, 2009). Given the rising global demand for a highly skilled workforce, governments have continued to make significant investments in higher education. For instance, the majority of the Organization for Economic Cooperation and Development (OECD) countries spent a larger percentage of their GDP on tertiary 
education in 2013 than they did in 2005 (OECD, 2017). Despite the continuing progress, however, $60 \%$ of employers consider recent graduates unprepared for the world of work, while $40 \%$ said a lack of skills was the reason for entry-level job vacancies (McKinsey Global Institute, 20172).

For smaller economies such as El Salvador, which have been based on the production of labor-intensive industries, increasing the number of high- and mediumskilled workers is critical if they are to remain competitive in the increasingly integrated global marketplace. Higher education, therefore, is integral to creating a skilled workforce, encouraging innovation and ultimately increasing economic growth prospects in El Salvador and other countries with economies dependent on labor-intensive legacy industries. While some may view the emphasis on labor market trends and skills development as antithetical to higher education's traditional mission, the authors take the position that building synergistic relationships between academia and the private sector strengthens social institutions, particularly in the context of developing countries plagued with stagnant growth, informal economies and violence, as in the case of El Salvador.

El Salvador has suffered from persistently low levels of growth $(2.3 \%$ over the past five years). Even with a steady increase in remittances, macroeconomic instability and low levels of investment have resulted in an inability to improve the country's competitive positioning in the region. El Salvador consistently ranks low in the Global Competitiveness Index of the World Economic Forum (WEF). For instance, under the skills pillar, El Salvador ranks 135 out of 140 countries in critical thinking, 108 in professional training and 112 in university programs oriented towards industry needs

2 This survey was done in nine countries (Brazil, Germany, India, Mexico, Morocco, Saudi Arabia, Turkey, the UK and the USA). 
(RTI International, 2019a). An often overlooked or undervalued factor has been the importance of human capital and skills in improving a country's competitiveness.

According to the US Agency for International Development's (USAID's) higher education assessment conducted in 2012, El Salvador's higher education institutions (HEIs) were not providing the quality of human capital required by industry to remain competitive and increase production, contributing to the near stagnant economy (Saunders et al, 2012). For example, its manufacturing industry cited the lack of appropriately skilled staff in quality control and good manufacturing practices as a major constraint to increasing productivity. The energy sector complained that qualified professionals were not available in the country, requiring them to pay more to bring skilled technicians from overseas. Many companies across sectors reported common problems in finding interns and new hires with workplace professionalism and realistic expectations, and soft skills such as critical thinking, problem solving and communication. On the supply side, students were enrolled in high numbers in traditional academic programs such as law, marketing, accounting and social services. In 2015, only 23\% of students enrolled in higher education were in STEM fields (Ministerio de Educación, 2016).

To address these challenges, in 2014 USAID initiated a \$22 million Higher Education for Economic Growth project in El Salvador - implemented by RTI International - to incentivize collaboration among HEIs, industry and government through industry-higher education clusters. The goals were to improve human capital, increase industry competitiveness and stimulate innovation in four sectors: information and communication technologies (ICT), energy and energy efficiency, light 
manufacturing, and agriculture and food production. Our contribution to this special issue on 'Higher education and workforce development' describes how the skills mismatch problem in El Salvador was addressed through this innovative model of collaboration between sectors that traditionally did not work together, ultimately resulting in new industry-linked programs to more adequately prepare students to succeed in the labor market.

\section{University-industry collaboration}

UICs have been covered extensively in the literature since the early 1990s. A comprehensive review by Ankrah and Al-Tabbaa (2015) identifies more than 1500 published articles on the topic, and analyzes 109 in depth. Companies that collaborate with universities often show gains in productivity and improved competitiveness when compared to companies that have lacked those collaborative relationships (Malairaja and Zawdie, 2008). Such results have spurred governments in advanced and emerging economies to enact polices aimed at stimulating collaboration in research and development, curriculum development, training for researchers in industry and knowledge sharing between industries and universities (Acworth, 2008; Salleh and Omar, 2013). However, less developed countries are not as well represented in the literature. A noteworthy exception is the study by Arocena and Sutz (2000), which examines national systems of innovation from the perspective of the South.

UICs are defined as rational, inter-organizational relationships that facilitate the exchange of resources (Ankrah and Al-Tabbaa, 2015). 'Resources' may be tangible goods such as materials and equipment, or intangible goods such as knowledge and 
research. In either case, industries and universities engage in collaborative relationships to expand access to resources that will benefit them (Koka and Prescott, 2002).

Barnes et al. (2002) identify the most salient characteristics of effective UICs. In the first stage, universities and industries that have complementary objectives and expertise are the best partners. Once formed, operationalizing the collaborative activities is the next stage of effective partnerships. The challenge at this stage is managing cultural differences between universities and industries. Meeting industry demands while maintaining a commitment to higher education's core mission can be daunting if not conflicting. Ensuring equality and mutual benefit that contribute to universal success, agreeing beforehand on outcomes, and monitoring environmental influence are essential for minimizing conflict and maintaining effective UICs.

Petrov et al. (2016) studied numerous cases a decade after the Leitch Report in the UK and found that, among the three stakeholders (industry, university and government funding agency), seven key factors enabled effective UICs for workforce development. According to the researchers, the seven factors are grouped into three stages. In the first, the formation of effective partnerships includes strategic fit, finding the right partners, and agreement on the learning needs. Operationalizing the partnership in the second stage requires identifying appropriate staffing, supporting a culture of change and reforming systems, and ensuring the necessary funding. Finally, persistence in sustaining and developing the partnership is necessary throughout, particularly when external funding ends.

Hordern (2014) similarly studied workforce development processes in HEIs that had resulted from policy changes and incentive grants stemming from the Leitch Report 
and identified three models. The first model of workforce development processes is the 'traditional disciplinary-based' educational program that arises from government, professional organizations or industry demands. The requisite knowledge and skills are often reflected in the curriculum and its learning objectives, which may be regulated by accreditation bodies or certification standards. The next two models are 'non-traditional workforce development processes' to engage industry and other stakeholders beyond the traditional disciplinary-based system. For instance, external industry experts may be invited to participate in what is the traditional domain of the faculty - to design the curriculum, sponsor the learning experience, and even co-teach the lessons. Workplace learning environments, internships and apprenticeships also play a prominent role in nontraditional processes. In the second model, external catalysts, such as funding and expertise, stimulate the change process while in the third model the change is limited to more discreet educational programs to meet individual industry needs.

Below we describe the development of UICs through the formation of industryhigher education clusters in El Salvador, which fits most closely into Hordern's second model of non-traditional workforce development processes. In addition, we analyze the cluster formation process according to the seven factors identified by Petrov et al. (2016) to facilitate and sustain effective industry-higher education partnerships.

\section{Model of change: industry-higher education clusters}

Developing industry-higher education alliances, or clusters (see Figure 1), was at the core of the USAID project's model of change. The clusters, made up of HEIs, industry and government, provided a platform for implementing the project's activities and were designed gradually to become self-sustaining. In El Salvador, the formation of the 
clusters took on an even greater importance in facilitating dialogue among individuals who were not only from different sectors but were also on opposite sides of a long and violent civil war.

The objective of the clusters was to foster collaboration on investments in workforce development and applied research projects. The grouping of universities and the private sector has reduced long-standing distrust between important players in Salvadoran society and has enabled key actors to value the investments in creating a collaborative platform to improve El Salvador's economic future. Through this model, private sector associations, employers, HEIs and government worked together as equal partners to educate students in the competencies required for professional and technical careers in high-growth fields, as well as to develop scientific and technological solutions to industry challenges.

\section{Figure 1. Industry-higher education cluster model.}

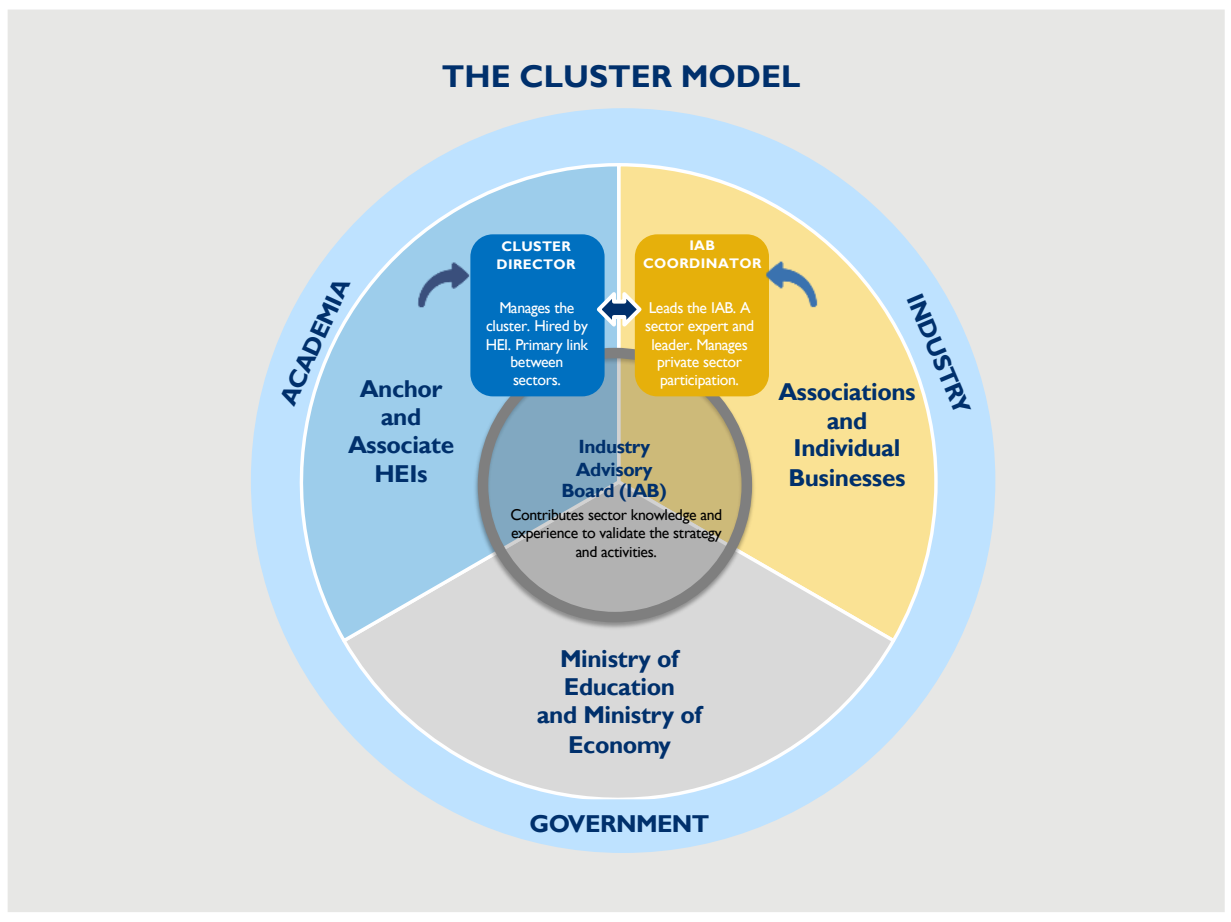

Source: USAID Higher Education for Economic Growth project 
In the economics literature, clusters constitute an array of linked industries and other entities that enhance the competitiveness of industry (Porter, 1998). Their contribution to economic growth lies in the relationships, knowledge and innovation that may be developed, which distant rivals cannot match, thus creating enduring competitive advantage in a global economy. Efforts to develop industry clusters increasingly include HEIs as central assets. HEIs provide research and technology, support the development of entrepreneurial skills and, most importantly, prepare a qualified workforce. The HEIs with the greatest impact are those with a robust base of research and/or education and technical training programs that are closely aligned with industry's demand for talent. Thus, industry-higher education clusters constitute an important strategy for closing skills gaps and developing a high- and medium-skills workforce in strategic fields for economic growth. This view of universities' role in society does not depart from their core mission, but broadens their mission to meet the needs of a changing society, much as the introduction of land-grant universities did in the USA.

\section{Forming, operationalizing, and developing clusters}

Similar to Petrov et al. (2016), the process facilitated by the USAID project of forming, operationalizing and sustaining industry-higher education clusters was divided into three phases of activity: Phase One - forming clusters, Phase Two - operationalizing clusters, and Phase Three - implementation and sustainability. Using clusters as a strategy to align industry and higher education to enhance workforce development adds to the framework for 'engagement activity' articulated by Petrov and colleagues (2016). Whereas they enumerated seven steps, the project developed a ten-step process, which included transparent, data-driven decision making and an emphasis on inclusiveness based on the 
commitment to a collaborative mindset to establish and sustain effective UIC. Figure 2 illustrates the 10 steps followed across the three phases of the cluster formation process. All three phases are briefly described in the following subsections.

\section{Figure 2. Steps for establishing clusters.}

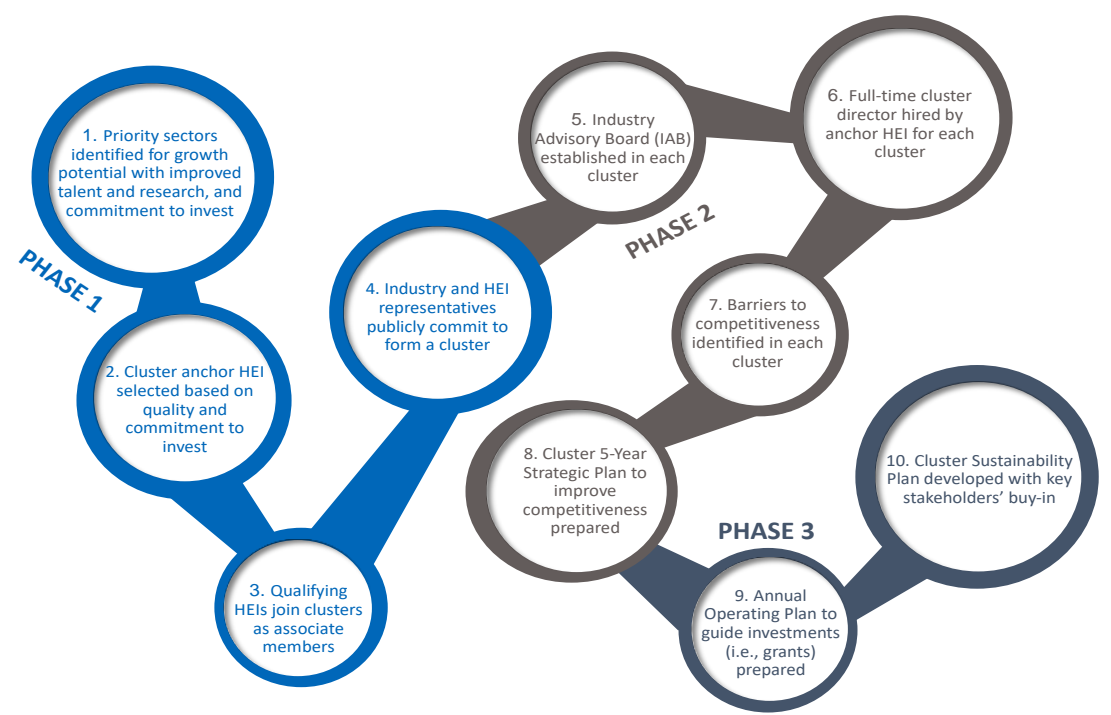

Source: Higher Education for Economic Growth project team

\section{Phase One: formation of clusters}

Phase One in El Salvador was characterized by the selection of high-priority economic sectors and their higher education partners, culminating in a high-profile, public commitment to collaborate as co-equal cluster members. The selection processes differed for the selection of priority sectors, industry partners and higher education partners. However, in each case the process was data-driven and transparent and adhered to a principle of inclusiveness for HEIs that sought to improve quality. From the beginning, addressing industry needs for improved human capital and applied research was the driving force or purpose for the formation of the clusters. 


\section{Step 1: Selection of dynamic economic sectors and industry champions}

The starting point was to identify high-growth and dynamic economic sectors and pick a select number to focus on for creating industry-higher education clusters. The project conducted a two-step process based on (1) economic criteria, using traditional economic analysis methods to selecting priority sectors; and (2) industry criteria, using an innovative approach to gauge which industries would be most willing to commit to achieving the objectives of the industry-higher education collaboration model and contribute time and resources.

The economic analysis identified sectors with the greatest potential for growth based on past and projected performance; a potential for increases in employment; competitiveness in export markets; a strong competitive position based on national and regional markets; and those already selected as specific priority sectors for the country by the government of El Salvador, as outlined in its 2014 Productive Promotion and Diversification Policy. In addition to analysis of the national and international economic data, project researchers interviewed industry associations and other private sector representatives to incorporate first-hand industry feedback on which sectors were most dynamic and poised for growth.

The next step in the industry selection process was to pick industry champions based on commitment criteria. The objective of this step was to measure the interest and willingness of industry leaders to collaborate with HEIs on improving their competitive potential through joint investments in talent and research. One of the challenges was to obtain up-front commitments from private sector partners, short of requiring specific financial contributions as a condition for becoming a formal partner. Hence, a structured 
approach was developed to gauge industry support through alternative examples of commitment.

The project developed a 'commitment index' along two dimensions. The first dimension was expressed willingness among industry groups to participate in some of the project's key areas of activities: (1) curriculum reform and advisory role on industry advisory boards (IABs), (2) internships (faculty externships and student internships) and (3) applied research. The second dimension included an analysis of the following factors: (1) industry interest (willingness to participate in project key activities), (2) existing projects/collaboration with HEIs (evidence demonstrating joint work with HEIs), (3) the prospect of funding and (4) the relevance of barriers that restricted the industry's competitiveness and the ability of the project to address those barriers.

The data-driven and transparent approach to selecting industry partners was important because there was significant private sector interest. Thus, the decision making as to which sectors and industry partners the project would focus on needed to be backed up by an objective rationale that both selected and non-selected industries could support. The approach instilled credibility in the process and resulted in buy-in from the private sector. It also allowed the project to gauge and measure industry's potential 'skin in the game' without asking for cash contributions too early in the relationship-building process.

\section{Step 2: Selection of HEI leaders for each cluster ('anchor HEIs')}

Recognizing that no one HEI had all the resources needed to meet industry requirements, the industry-higher education clusters engaged multiple HEIs to collaborate in responding to industry. Furthermore, one university in each cluster was selected to serve 
as the leader, or the 'anchor HEI'. HEIs could serve as associate members in more than one cluster, but could serve as the anchor of only one.

As indicated earlier, the selection process for identifying HEI partners was based on principles of transparency, objectivity and rigor using data-driven decision making. A strong emphasis was placed on HEIs that demonstrated a high level of commitment to partnering, were open to reform, were positioned to capitalize on the assistance provided through project resources with investments of their own, and were ready to fully commit to the shared objectives of the project. Another guiding principle in the selection of HEIs was the involvement of industry partners in the decision-making process, allowing the private sector to have a consultative role in determining which HEIs were most prepared to meet the needs the respective industry sectors.

\section{Step 3: Formation of broad alliances among HEIs ('associate HEIs')}

The clusters emphasized inclusivity as an important aspect of the model, giving all HEIs an opportunity to participate as 'associate HEI' members if they were unable to serve as an anchor institution. This model of collaboration across multiple HEIs helped to strengthen social capital across the higher education sector. HEIs participating as associate members benefited from cluster activities in faculty training, curriculum upgrades and applied research. As part of the cluster, they also benefited from learning and knowledge sharing.

Associate HEIs were institutions that demonstrated some capacity for research and training and were committed to work collaboratively with other HEIs. Among the 40 HEIs in El Salvador, none on its own represented all the capacity needed to meet the needs of industries in each cluster for talent and research. However, by working together, 
HEIs could pool their resources and capabilities to better meet industry's needs. This approach required a shift in mindset away from competition and toward collaboration, thus creating an alliance of HEIs to achieve synergies to serve the common good of the sector and of the country's higher education system.

Crossing boundaries, building trust and investing in cooperative initiatives were necessary in the first stage of forming clusters and remained essential in the second stage of operationalizing them.

\section{Step 4: Bringing industry and higher education together and launching}

\section{clusters}

By the end of the project's first two years, four clusters had been established with 12 of the 40 HEIs in El Salvador as HEI associates. 3 Together, these clusters brought together academia and industry through significant representation on each side. The 12 HEIs enrolled more than $90 \%$ of the STEM higher education students in the country (Ministerio de Educación, 2016) while the four industry associations represented more than 400-member companies.

The culmination of Phase One was the high-profile, public commitment of industry and HEIs to collaborate in forming the four clusters. In a post-conflict country such as El Salvador, this act was highly symbolic of reconciliation and a commitment to re-establish trust between the private sector, which had been aligned with the political right, and the academic sector, which was characterized as aligning with the left. After almost 25 years since the end of the civil war, the two sides came together for the betterment of the country. It is perhaps for this reason that the launch of each of the 
clusters was a major public event. Alongside industry and higher education leaders, senior Salvadoran government officials participated in all four launch events and the US Ambassador participated in three. The launch of the manufacturing cluster was organized in parallel with an innovation summit to generate dialogue between industry, higher education and government on innovation and research collaboration. The 'buzz' created by high-level dignitaries enhanced the enthusiasm and interest in participating in the clusters.

\section{Phase Two: operationalizing the clusters}

Phase Two of the cluster formation process involved establishing the organizational structure in which decisions for investment would be made. In Petrov et al.'s (2016) formulation, operationalizing the partnership involved finding the right staff, supporting a culture of change and reforming systems, and ensuring funding. In the case of El Salvador, operationalizing the clusters also involved setting up the structures for shared decision making that were necessary for the continuing development of trust and understanding in the collaborating organizations of each other's worlds, the identification of barriers to competitiveness, and the formulation of strategic plans and annual operating plans to increase productivity, innovation and economic growth.

\section{Step 5: Establish an Industry Advisory Board (IAB) for each cluster}

The project facilitated the creation of El Salvador's first-ever IABs in higher education and industry. Before, Salvadoran universities lacked liaisons who focused on understanding industry needs, building relationships with the private sector and coordinating educational planning with industry representatives. The IABs were a mechanism to encourage university and industry partners to actively step into each 
other's world, to communicate regularly and jointly plan how to better align education with industry.

Across the four clusters, the IAB membership was formed with industry associations, high-level industry representatives and business professionals, HEI officials (a mix of institution presidents and curriculum coordinators), and representatives of government agencies. As advisory partners, industry representatives and higher education leaders worked together to ensure that HEIs stayed current with industry trends, employer hiring practices and skills needs for entry-level talent. Sharing this type of labor market information on a regular basis helped guide decision making at HEIs in launching new degree or certificate programs, making curriculum changes, developing internship programs and improving student career services, among other areas. For competencybased curricula, employers also defined the specific competencies and skills required for respective occupations and majors.

\section{Step 6: Appoint a dedicated cluster director}

The industry-higher education cluster model required a dedicated (full-time)

management structure to effectively implement cross-sector collaboration and oversee the complex relationships among HEIs, industry associations, private sector companies and government stakeholders. Each cluster was managed by a cluster director who was appointed and financed by the anchor HEI for each cluster, ensuring a model of sustainability and local ownership.

Ideally, the cluster director was a senior member of the anchor HEI faculty, had the confidence of the institutional leadership and had an established track record of research in collaboration with industry and in mentoring students who were well placed 
in industries. The profile of an ideal cluster director was not necessarily that of a successful academic by traditional academic standards. The entrepreneurial faculty member was often the outlier among his or her colleagues, and was highly valued by the industries represented in the cluster.

The industry-higher education cluster also demanded that the director represented the whole cluster and not just the anchor HEI, even though their compensation and academic home were determined by the institution. Because multiple institutions were cooperating in the cluster, the director had to become very familiar with the human and physical resources of the associate HEI members as well. In other words, collegiality had to extend beyond institutional boundaries to include all cluster members.

\section{Step 7: Identify barriers to cluster competitiveness}

Before developing a strategy to enhance cluster competitiveness, each of El Salvador's industry-higher education clusters commissioned a competitiveness profile to identify and analyze barriers to industry competitiveness within each cluster. These cluster profiles analyzed the recent performance of each of the four sectors and identified economic growth drivers and workforce development trends for a clearer understanding of barriers that were inhibiting growth and productivity, areas of comparative advantage, and opportunities to enhance competitiveness. The researchers conducted an economic analysis and key informant interviews with leaders in industry, academia and government. They also used the SWOT analysis framework to identify overall strengths, weaknesses, opportunities and threats for each cluster. 


\section{Step 8: Collaboratively develop a common vision through cluster strategic}

\section{plans}

Persistence in developing and sustaining the partnerships was aided by developing competitiveness strategic plans. The cluster strategic plan represented a vision for how firms and educational institutions could collaborate across sectors to achieve growth. It also represented a vision for how firms could work with other firms and university members with their other academic peers, rather than seeing one another as competitors.

When competitiveness strategies were being designed, it was very important to involve stakeholders at all levels of the cluster in a participatory manner so that all perspectives were incorporated and the different actors in industry, higher education and government felt a sense of ownership of the vision and strategy. To this end, cluster members attended two-day strategic planning workshops. During the first day the participants reviewed the previously prepared cluster competitiveness profile, which identified obstacles such as skills gaps due to a lack of understanding of industry requirements, outdated curricula and the need for more applied research. Members of each cluster discussed their visions for how to strengthen the competitiveness of their respective sectors, how to improve the position of the industry in the regional or global context if relevant, and how to develop the talent pipeline needed to take the industry forward.

On the second day, members developed strategic initiatives to overcome barriers and increase industry competitiveness. Although most of the participants had experience with strategic plans in their respective companies or educational institutions, no one had ever participated in strategic planning for an industry-higher education joint effort to 
make the sector as a whole competitive. One significant outcome from the joint planning session was that each cluster identified a strategy to sustain the cluster beyond the life of the project.

Dialogue became the critical element for each cluster to see itself as an integrated system, a process that was enhanced in the third phase of engagement activity.

\section{Phase Three: implementation and sustainability}

Phase Three of the cluster formation model was 'where the rubber hit the road' through ongoing cluster dialogue and collaboration to develop annual work plans, implement joint activities and invest in sustainability planning. This followed a holistic, demand-driven process, in which the IABs advised and provided strategic vision, the cluster directors synthesized strategy and provided leadership, and cluster working groups were formed to implement specific activities.

While the process is presented linearly, in practice, several activities occurred simultaneously. In some cases, curriculum reform or faculty training activities began before strategic plans were completed and integrated into annual work plans. This allowed for 'quick wins' alongside the slower-moving process of building trust and developing the structures of cluster partnerships.

\section{Step 9: Develop annual work plans and implement activities}

While the cluster strategic plans created the 'big picture' vision for aligning higher education programs with industry priorities, work plans were needed to provide a roadmap on how to implement concrete activities. Based on the strategic priorities identified in the five-year cluster strategic plan, each cluster developed an annual work plan to define implementation actions, focus activities, set timelines and guide investment 
priorities. In general, each cluster developed work plans that included the following types of higher education and workforce development activities: curriculum reform, the development of internationally recognized certificate programs, the establishment of faculty externships in industry to bring real-world experiences into the classroom, the establishment of career centers, and student participation in internships or industry applied research projects.

The project included a $\$ 5$ million grant program, with a 1:1 match from HEIs and the private sector. Requiring that resources be leveraged was critical for the joint ownership of activities, especially as it related to curriculum implementation and applied research. In many ways, the grants program provided the needed incentive for universities to work hand-in-hand with the private sector in order to deliver industrylinked education.

\section{Step 10: Planning for sustainability}

Although the clusters are at an early stage of their life cycle, sustainability was explicitly addressed as part of the formation process. Benchmarking clusters with global practice and giving key stakeholders the opportunity to have shared experiences provided the additional ingredients necessary for commitment to a long-term vision. At the end of 2016, a high-level 12-member delegation of the project's key stakeholders - representing anchor universities, industry associations and the Salvadoran government - participated in a pivotal study tour to Research Triangle Park in North Carolina, USA. During this visit, the stakeholders were able to dig deeper into how sustained partnerships transformed the state into a model of economic development, innovation and growth. They understood that the success of the model was built on a commitment to long-term 
collaboration and mutual respect for the role each side played. The visit also provided opportunities for formal and informal exchanges among the group, which was paramount in solidifying their new relationship.

Benchmarking against global practice shielded the clusters from their own fragility and encouraged stakeholders to strengthen their commitment to the cluster model approach and more generally toward collaboration. The experience also underscored the need for and interest in a strategic steering committee composed of HEIs and industry across the four clusters, along with the participation of both the Salvadoran Ministry of Economy and the Ministry of Education. One of the key areas the steering committee prioritized was how to sustain the clusters in the long term. Between 2017 and 2018, the clusters participated in developing a sustainability plan assessing both global best practice and the local context in El Salvador. The plan prioritized the neutrality and independence of the clusters, which has enabled trust among stakeholders. The plan also recognized the structures already in place that could serve as the foundation for the future: those include the IABs, the cluster directors and the steering committee, as well as cluster members. In early 2019, cluster stakeholders, especially HEI presidents and industry association leads voiced their commitment to the cluster model resulting in the establishment of the Consortium of Higher Education Institutions for Economic Growth, an independent organization that will oversee activities across the four clusters (RTI International, 2019b).

\section{Discussion}

Our contribution adds to the literature demonstrating that industries which collaborate with higher education institutions to invest in human capital development can become 
more productive and innovative. HEIs provide research and technology, support the development of entrepreneurial skills and, most importantly, prepare a qualified workforce. However, as noted by Barnes et al. (2002), managing the cultural differences between industry and higher education actors is one of the biggest challenges in developing constructive relations. The case of the USAID project in El Salvador demonstrates that clusters are an effective strategy for bringing industries and HEIs together to form sustainable partnerships for the purpose of closing the skills gap and developing a high- and medium-skills workforce in priority areas. The case contributes to our understanding of the process of forming, operationalizing and sustaining effective UIC partnerships, particularly in small and developing economies.

\section{Developing sustainable partnerships}

The initiative to promote higher education-industry collaboration in El Salvador was not endogenous, but came about as a result of an external catalyst in the form of US foreign assistance. Similar external catalysts have appeared in other forms, such as the response to the Leitch Report in the UK and President Obama's call to increase the number of Americans with college degrees. In all three cases, external funding was tied to disrupting the status quo, promoting a change mindset leading to systemic reforms that resulted in enhancing the role of HEIs in the value chain of industrial productivity, which, according to Arocena and Sutz (2000), is particularly relevant to establishing national systems of innovation in developing countries.

Petrov et al. (2016) identified three key factors in the partnership formation stage: strategic fit, finding the right partners and agreement on learning needs. As we describe in the El Salvador case, the strategic fit was present from the start as government, higher 
education and industry leaders agreed on the broader challenge that a lack of skilled human capital was inhibiting the country's economic growth. However, the process required a significant investment of time to bring together different sectors which traditionally had not worked with each other. While private sector associations and HEIs showed initial enthusiasm in working with the project's model of change, with respect to managing the cultural differences it took time to work out the details of the cluster partnership regarding roles, responsibilities and the terms of the partnership.

While the main focus was on finding the right partners in the academic and private sectors, the government played an important symbolic role in supporting the formation of the industry-higher education clusters at the onset through active consultation from the Ministry of Education and the Ministry of Economy. Over time, the government remained engaged by participating in each of the IABs, participating in cluster launches, facilitating dialogues and approving curriculum changes.

Once the clusters were formed, operationalizing them posed a new set of challenges that demonstrated key factors in the second stage: appropriate staffing, supporting a culture of change and reforming systems and ensuring adequate funding (Petrov et al., 2016). The cluster director and IAB chair were two leadership roles that were essential in getting the cluster going. Effective leadership required individuals who were not only respected within their sector but were also experienced in crossing boundaries and bringing together diverse perspectives to achieve common objectives, as well as having demonstrated an innovative disposition. Most importantly, effective leadership required individuals who were committed to a better future for their country, not just for their business or university. 
While the \$22 million in USAID funding was the catalyst for bringing industry and university partners to the table, the project made clear in its commitment criteria that participation meant committing additional resources. Anchor universities had to make the largest up-front commitments as a part of the selection process, but industry partners also had to invest initially their time and later their resources to support applied research and curriculum development. Contributions were gradual or came only after some period of engagement. Producing early wins was necessary for some private sector partners to overcome their hesitancy to contribute financially, but once they could see concrete benefits they started to contribute resources. For instance, when the final round of applied research projects was completed, HEIs were able to secure leverage of more than $\$ 0.5$ million from 26 different businesses. Regardless of timing, it was important to set the expectation that all partners were expected to show 'skin in the game', with real commitment to resources.

Crossing boundaries, building trust through a transparent data-driven selection process and investing in collaborative activities were the essential elements in the third stage of what Petrov et al. (2016: 94) referred to as 'persistence in sustaining, developing and leading the partnership'. Each cluster developed differently, a process that was driven by the realities in the implementation phase. Stakeholders came to the table with their priorities, as well as their current and ongoing commitments. Dialogue among stakeholders was necessary to manage differences in expectations and served to expose their priorities and commitments and to identify opportunities to harmonize them. One way to manage the culture gap among participants was by encouraging educators and industry leaders to step into each other's world by meeting frequently, visiting each 
other's work environment, and using common vocabulary. For instance, HEIs would often present information on their academic programs and course content to industry representatives during the IAB meetings because they found that companies were often simply not aware of the breadth of their existing educational programs.

Opportunities for interaction among individuals and institutions within the cluster were frequent and repeated, which strengthened the social capital and networks that would be required over the long term. One unexpected outcome of the clusters was the close working relationship that developed among the four anchor HEI presidents. The presidents, who did not know each other beforehand, now consider themselves friends. Through the cluster model and related project activities, HEI leaders were pulled into frequent interactions with their peers around important topics of higher education policy, scholarships, curricula reform, research and career services. They recognized the power of their influence through coordinated action.

Creating a common vision was necessary to support a change mindset. However, that common vision, while necessary, was not sufficient. The cluster competitiveness profiles and the participatory strategic planning helped to achieve a common framework to identify the problem and develop solutions. Actors can unite around a common framework and shared understanding. A roadmap or work plan that moves the group forward with clear direction, roles and responsibilities, a timeline, and actionable steps was also necessary. Working groups needed to have clear goals, deadlines and clearly defined responsibilities to keep everyone on track and motivated.

The cluster model allowed industry and higher education partners to define what the 'success' of the collaboration would look like together. Equally important were the 
mechanisms and indicators that clusters developed to keep themselves accountable to each other. Success indicators were designed to measure the results of the collaboration for example, how industry's satisfaction with the quality of skills and applied research being developed by HEIs would change over time. Another success measure examined the sustainability of structures, such as the IABs, created within the cluster model: whether they would last or how they would evolve beyond the support of donor funding.

\section{Examples of cluster-related disruption}

We believe that the cluster model has effectively disrupted the status quo, particularly the norms surrounding industry-higher education cooperation for workforce development. Industry and higher education have traditionally come together to update disciplinary training or to meet individual industry needs, which does little to change the fundamental relationship between the two sectors. However, the industry-higher education cluster experience in El Salvador most closely fits into Hordern's (2014) second model of nontraditional workforce development processes in which industry involvement in curriculum development and workplace learning environments (such as internships, student involvement in applied research and faculty externships) plays a prominent role in disrupting academia's traditional disciplinary-based system. Four years of university and industry collaboration through the cluster experience has led to a fundamental change in industry-higher education relations, which we anticipate will extend well beyond the life of the USAID project. The following are examples of how the clusters in El Salvador have improved industry-higher education relations.

Clusters facilitated the process of making curricula relevant to labor market needs through meaningful engagement with industry. In the energy and energy efficiency 
cluster, Sherwin Williams and the US Green Building Council collaborated with the anchor university to upgrade eight engineering degrees with a new course on green building design. The resulting LEED (Leadership in Energy and Environmental Design) Lab is the first of its kind in Central America and provides teachers, students and businesses with hands-on practical learning for the design of sustainable and energy efficient buildings. By working with industry partners in curriculum design, the university became the first HEI in Central America to prepare professionals with multidisciplinary skills in green design and construction.

Meaningful engagement with industry in the ICT cluster resulted in six HEIs coming together to revise their outdated IT curricula based on workforce needs identified in the competitiveness profile. The norms governing relationships between HEIs were disrupted as the six HEIs worked together and with industry partners to develop a common computer science curriculum that incorporated up-to-date discipline knowledge, new technology and industry trends. Not only were students guaranteed similar learning objectives and standards regardless of institution, they were also given access to four specializations during their elective years. Further changing institutional norms, the HEIs agreed among themselves which universities would deliver each of the specializations.

Applied research projects have provided more than a 100 students at 10 different universities not only with practical experience in conducting the fundamentals of research but, equally importantly, with experiential learning that has exposed them to real-world challenges faced by industries. In the agro-industry sector, students at the University of El Salvador helped research faculty grow and test new varieties of coffee and cacao. The goal was to help small producers increase their production and potential for export. 
Students learned experientially in the lab testing different plant varieties, but also by engaging directly with the market producers, delivering technical demonstrations to farmers interested in the commercial production of coffee and cacao. Through this experience, the students earned a distinct professional profile that set them apart from their peers. Their practical skills also make them more employable, given their potential to be immediately productive professionals in the growing cocoa and coffee industries in El Salvador and the region.

Finally, the creation of career development centers (CDCs) in universities has helped to bridge the gap between education and work. Prior to the CDCs, career support was at best ad hoc. With the project's support, five universities have established fully functioning CDCs, including support for a private sector partnerships coordinator who initiates and maintains strategic partnerships with employers. CDCs are strengthening the link between the HEIs and future employers, and improving the transition from education to employment for students, particularly in STEM fields. Work-based experiential learning and even job opportunities for students increased when CDCs were able to directly engage with employers in strategic partnerships rather than one-off interactions.

\section{Conclusion}

The industry-higher education clusters have validated HEIs as key actors in advancing El Salvador's workforce development and competitiveness goals. Traditionally, HEIs have not even had a seat at the table when important discussions have taken place about employment, economic growth, productivity, markets and innovation. Their propensity to look inward has also created large gaps between the education they provide and the skills most needed by the market. But the status quo is shifting. The cluster platform provides 
opportunities for universities to demonstrate their value in the workforce development ecosystem as the main suppliers of higher-skilled human capital and research. This is especially important in the Fourth Industrial Revolution, in which a premium will be placed on individuals and innovations that can add value. What is more, because of the manner in which clusters were formed - through data-driven, transparent and inclusive processes - there have been more trust in the model and willingness to take risks, which developed into ownership on the part of the key stakeholders. Finally, because collaboration and sustainability were emphasized from the onset, the clusters are poised to evolve well beyond the USAID project.

Further research to add to the literature in this field could assess the impact of university-industry collaboration on workforce development outcomes; for example, the ease of finding skilled employees. Another important topic deserving further exploration is the extent to which organizational development changes are necessary for universities to become more agile organizations, able to pivot to become outward-facing institutions. The role and qualities of leadership in both the university and industry sectors to develop effective partnerships, as well as the link between curriculum and labor market outcomes, would be fruitful fields of inquiry.

Finally, for practitioners and policy makers, the industry-higher education cluster model has potential for application in other contexts, particularly in developing countries that lack the requisite talent and resources in a single HEI to meet industry demands for a high- and medium-skills workforce to increase competitiveness. Looking to the future in the context of the Fourth Industrial Revolution and its disruptive trends, the links between industry, academia and government will be more important than ever to produce human 
capital that is skilled, has the capacity to learn and relearn, is resilient and is endowed with socio-emotional skills that are not susceptible to substitution by automation.

\section{References}

Acworth E (2008) University-industry engagement: The formation of the knowledge integration community (KIC) model at the Cambridge-MIT Institute. Research Policy 37(8): 1241-1254.

Ankrah S and Al-Tabbaa O (2015) Universities-industry collaboration: A systematic review. Scandinavian Journal of Management 31(3): 387-408.

Arocena R and Sutz J (2000) Looking at national systems of innovation from the South. Industry and Innovation 7(1): 55-75.

Barnes JL, Zarrugh MY, Lawrence DJ and McKown RL (2000) A governmentuniversity-industry response to critical manufacturing innovation needs. Industry and Higher Education 14(2): 73-91.

Barnes T, Pashby I and Gibbons A (2002) Effective university-industry interaction: A multi-case evaluation of collaborative R\&D projects. European Management Journal 20(3): 272-285.

Hordern J (2014) Workforce development, higher education and productive systems. Journal of Education and Work 27(4): 409-432.

Koka BR and Prescott JE (2002) Strategic alliances as social capital: A multidimensional view. Strategic Management Journal 23(9): 795-816.

Leitch S (2006) Prosperity for All in the Global Economy-World Class Skills, London: Department for Education and Skills. 
Malairaja C and Zawdie G (2008) Science parks and university-industry collaboration in Malaysia. Technology Analysis and Strategic Management 20(6): 727-739.

McKinsey Global Institute (2017) Technology, jobs and the future of work. Executive Briefing, May.

Ministerio de Economía (2014) Política Nacional de Fomento, Diversificación y Transformación Productiva de El Salvador. San Salvador, El Salvador.

Ministerio de Educación (2016) Resultados de la Información Estadística de Instituciones de Educación Superior. San Salvador, El Salvador: Dirección Nacional de Educación Superior.

OECD (2017) In-Depth Analysis of the Labour Market Relevance and Outcomes of Higher Education Systems: Analytical Framework and Country Practices Report. Enhancing Higher Education System Performance, OECD, Paris.

Petrov G, Southall J and Bolden R (2016) Making the 'third stream', mainstream: Facilitating effective higher education-employer engagement in workforce development. Higher Education Review 48(3): 75-99.

Porter, ME (1998) Clusters and the new economics of competition. Harvard Business Review 76(6): 77-90.

RTI International (2019a) USAID Higher Education for Economic Growth Activity: A Path Towards the Fourth Industrial Revolution. Achievements and Sustainability. Report for USAID El Salvador, Economic Growth Office. Contract AID-519-C-1400004.

RTI International (2019b) USAID Higher Education for Economic Growth: Final Report. Report for USAID El Salvador, Economic Growth Office. Contract AID519-C-14-00004. 
Salleh MS and Omar MZ (2013) University-industry collaboration models in Malaysia. Procedia: Social and Behavioral Sciences 102: 654-664.

Saunders R, Rivas, F, Rabossi, M et al (2012) El Salvador: Higher Education Assessment and Recommendations. Final report for USAID under Task Order AID-519-BC-1100001 of the Global Evaluation and Monitoring (GEM) II BPA, Contract EDH-E00-08-00003-00.

The White House (2009) The president addresses joint session of congress. 24 February 2009. 